\title{
Gastrointestinal toxic effects in patients with cancer receiving platinum-based therapy
}

\author{
Hamzah Abu-Sbeih¹, Niharika Mallepally², Ryan Goldstein², Ellie Chen², Tenglong Tang1, Uzoamaka K. \\ Dike $^{3}$, Mazen Al-Asadi ${ }^{1}$, Shannon Westin ${ }^{4}$, Daniel Halperin ${ }^{5}$, Yinghong Wang ${ }^{1 凶}$
}

1. Department of Gastroenterology, Hepatology, and Nutrition, The University of Texas MD Anderson Cancer Center, 1515 Holcombe Blvd, Houston, TX 77030, USA

2. Department of Internal Medicine, Baylor College of Medicine, 6620 Main St, Houston, TX 77030, USA

3. Department of Internal Medicine, McGovern Medical School at The University of Texas Health Science Center at Houston, 6431 Fannin St, Houston, TX 77030, USA

4. Department of Gynecologic Oncology and Reproductive Medicine, The University of Texas MD Anderson Cancer Center, 1515 Holcombe Blvd, Houston, TX 77030, USA

5. Department of Gastrointestinal Medical Oncology, The University of Texas MD Anderson Cancer Center, 1515 Holcombe Blvd, Houston, TX 77030, USA

$\square$ Corresponding author: Yinghong Wang, MD, PhD, Department of Gastroenterology, Hepatology and Nutrition, The University of Texas MD Anderson Cancer Center, 1515 Holcombe Blvd., Unit 1466, Houston, TX 77030. Tel: (713) 794-5073. Fax (713) 794-5398. Email: ywang59@mdanderson.org

(c) The author(s). This is an open access article distributed under the terms of the Creative Commons Attribution License (https://creativecommons.org/licenses/by/4.0/). See http:/ /ivyspring.com/terms for full terms and conditions.

Received: 2019.06.20; Accepted: 2019.11.14; Published: 2020.03.04

\begin{abstract}
Background: Platinum-based therapy (PBT) can be limited by gastrointestinal adverse events, particularly PBT-related colitis and diarrhea (PCD). We studied clinical features, treatments, and outcomes of PCD.

Methods: This was a retrospective study of cancer patients who received PBT and colonoscopic evaluation for PCD symptoms from 2009 to 2018.

Results: Of 36,595 patients who received PBT, 86 (0.2\%) met inclusion criteria. Median time from PBT initiation to PCD was 66 days. Regarding PBT type, $47 \%$ of the patients received carboplatin, $31 \%$ cisplatin, and $22 \%$ oxaliplatin. Median duration of PCD symptoms was 20 days. Colonoscopy revealed mucosal ulceration in $34 \%$ of the patients and nonulcerative inflammation in $33 \%$. Half of the cohort needed hospitalization for PCD (49\%). The majority received treatment for PCD (59\%): immunosuppressive therapy in $21 \%$, antibiotics in $27 \%$, antimotility agents in $22 \%$, and intravenous fluids in $51 \%$. Eight patients (9\%) were admitted to the intensive care unit for PCD management. Six patients (7\%) experienced colonic perforation that required surgical intervention; two of them had gastrointestinal tumors. Physicians restarted PBT in 37 (43\%) patients; 8 (22\%) of them had PCD recurrence that was managed expectantly. Colonic perforation occurred more frequently with use of oxaliplatin and cisplatin than carboplatin $(P=0.05)$. The median duration of $P C D$ symptoms was longer in patients receiving carboplatin or cisplatin than in those receiving oxaliplatin $(P=0.182)$.

Conclusions: $P C D$ is rare, but in a small subset of patients, it can lead to serious complications. Treatment of PCD is mainly supportive, but immunosuppressive therapy may be required.
\end{abstract}

Key words: platinum, carboplatin, cisplatin, gastrointestinal toxicity, colitis

\section{Introduction}

Platinum-based therapy (PBT) includes that with carboplatin, cisplatin, or oxaliplatin and is used for a wide variety of malignancies, including ovarian, lung, head and neck, and rectal cancers; neuroblastoma; and many others [1]. Because these agents have been in use since the early 1980s, they have a well-established efficacy profile and are on the World Health Organization's list of essential medicines [2]. Structurally, carboplatin and cisplatin are analogs of one another and have similar active metabolites. Functionally, these three agents have slightly different mechanisms of action. Platinum-based antineoplastic 
agents serve as DNA adducts that cause DNA proteins to cross-link. This cross-linking inhibits further DNA synthesis and repair in malignant cells $[1,3]$.

As reported in the literature, platinum agents are mainstays in lung cancer chemotherapy, often as first-line treatment. Studies have also supported the use of PBT for ovarian, cervical, uterine, and colorectal cancer [4-8]. In short, the applications of PBT are broad and well documented. Nevertheless, the utility of PBT has been impacted by its side effects. Platinum agents have dozens of reported side effects, some of which differ according to the agent. Specifically, oxaliplatin can be neurotoxic, carboplatin can cause myelosuppression, and cisplatin can be both nephrotoxic and neurotoxic. Use of all three agents has been associated with bone marrow toxicity, hepatotoxicity, mucositis, and, most notably, abdominal complaints. A review of treatment with cisplatin documented its ability to create electrolyte imbalances [9]. For currently used platinum agents, side effects are often dose-dependent and require monitoring using serial laboratory tests.

The literature on the physiology, efficacy, and general adverse effects of PBT is comprehensive. However, virtually no studies have focused on colitis related to use of these agents. Given that PBT can last for months and is given to potentially debilitated cancer patients, understanding PBT-related colitis and diarrhea (PCD) is essential. Therefore, we performed the study described herein to identify and characterize platinum-related colitis.

\section{Methods}

\section{Patient Selection and Data Collection}

After obtaining approval from the Institutional Review Board at The University of Texas MD Anderson Cancer Center, a retrospective, descriptive, single-center study of cancer patients who underwent PBT was conducted. Specifically, patients who received PBT with oxaliplatin, carboplatin, or cisplatin and underwent endoscopy along with biopsy analysis from 2009 to 2018 were screened. Patients in whom colitis symptoms developed following PBT and who underwent colonoscopic evaluation with biopsy analysis for PCD were included. PBT could have been given in a single-agent regimen or as part of multiagent one, as neoadjuvant or adjuvant treatment, and under a clinical trial or otherwise. Patients with competing etiologies of colitis, such as graft-versus-host disease and infectious colitis, were excluded.

\section{Clinical Characteristics}

After the study cohort was identified, their data were extracted from institutional electronic medical records. The data included demographics (age, sex, and race/ethnicity), medical and oncological history, medical comorbidities included in the Charlson Comorbidity Index,[10] smoking status, and recent nonsteroidal anti-inflammatory drug or aspirin use (within the previous 3 months). Oncological history was screened for variables related to 1) cancer type, 2) chemotherapy regimens (agents were listed individually), 3) noncolitis adverse events, 4) resumption of PBT after resolution of colitis, and 5) radiation therapy delivered to the abdominal region.

\section{Clinical Presentation and Treatment of PCD}

Clinical symptoms and signs of PCD in the study patients were diarrhea, abdominal pain, nausea and vomiting, blood or mucous in stool, and concurrent fever. At the time of PCD onset, findings on computed tomography (CT) scans suggestive of colitis included mucosal enhancement, colonic wall thickening, and pericolonic fat stranding. The presence of bacteremia or a gastrointestinal infection at the time of PCD development was documented. Of note, treatments of PCD symptoms were recorded qualitatively and included supportive care, antimotility agents, pain medications, intravenous fluids, antibiotics, immunosuppressive therapy, and surgery.

\section{Endoscopic and Histological Evaluation}

The patients' colonoscopy and sigmoidoscopy data were also retrieved. Examined variables included features of ulcers, the presence of erosion, the patterns and locations of inflammation (e.g., erythema, friability, nodularity, loss of vascular pattern, edema), and the presence of exudates. Histological records were examined for signs of acute inflammation, such as neutrophilic infiltrates, cryptitis, crypt abscesses, apoptosis, and eosinophilia. Chronic inflammation patterns were also noted, including lymphocytic infiltrates in the lamina propria, crypt architectural distortion, Paneth cell metaplasia, and granuloma or generalized chronic active inflammatory patterns. Microscopic colitis features with lymphocytic or collagenous patterns in the epithelium were also noted.

\section{Clinical Outcome}

Clinical outcomes of PCD examined in the patients' charts included need for admission to the hospital or intensive care unit (ICU); duration of hospitalization, gastrointestinal symptoms, and PCD treatment; PCD-related colonic perforation; recurrence of gastrointestinal symptoms after improvement; repeated endoscopic evaluation; and death. 


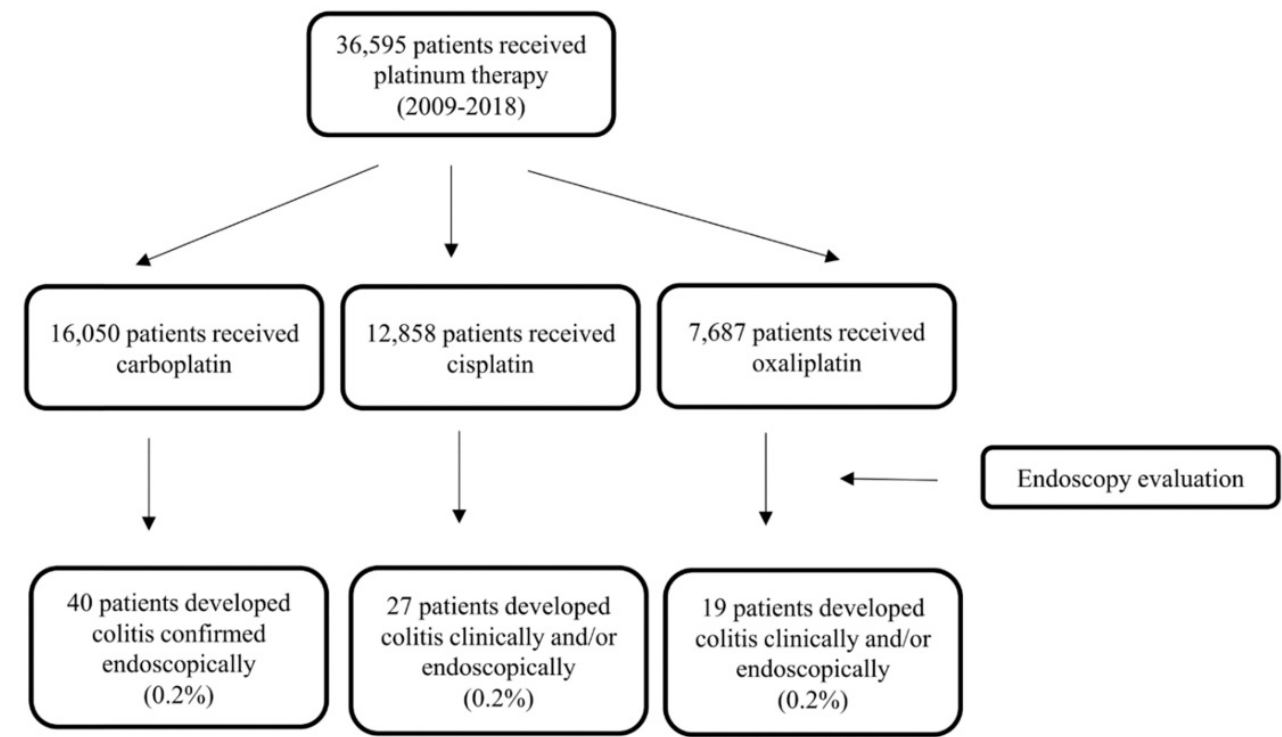

Figure 1. Flowchart of the study patients.

\section{Statistical Analysis}

Statistical analyses were carried out using the SPSS software program (version 24.0; IBM Corporation, Armonk, NY). Categorical variables were summarized using frequencies and proportions. Continuous variables were presented as medians and interquartile ranges (IQRs) or means ( \pm standard deviations). The Fisher exact and chi-square tests were used to compare categorical variables. The Wilcoxon rank sum test was used to compare continuous variables. All statistical tests were two-sided. $P$ values of up to 0.05 were considered statistically significant.

\section{Results}

\section{Patient Characteristics}

Of 36,595 patients who received PBT from 2009 to $2018,16,050$ received carboplatin, 12,959 received cisplatin, and 7,687 received oxaliplatin. A total of 86 $(0.2 \%)$ patients had PCD confirmed via endoscopy (Fig. 1), thus meeting the inclusion criteria for our study. Their median age was 57 years (IQR, 49-66 years). The population's clinical characteristics are outlined in Table 1 . Only $11 \%$ of them had preexisting inflammatory bowel disease (IBD), among them, 4 had active disease activity requiring medical treatments. The most common primary malignancy was gastrointestinal tract cancer $(28 \%)$ followed by lung (21\%), gynecological $(19 \%)$, and genitourinary $(11 \%)$ cancers. Almost half of the study participants received carboplatin (47\%), with $31 \%$ receiving cisplatin and $22 \%$ receiving oxaliplatin. The median duration of PBT was 51 days (IQR, 21-114 days).

\section{Clinical Presentation and PCD Treatment}

PCD symptoms developed in the participants at a median of 66 days (IQR, 9-153 days) after starting PBT. During initial presentation, the most common clinical symptom was diarrhea (62\%). Abdominal pain and blood or mucous in stool were also common, occurring in $48 \%$ and $43 \%$ of patients, respectively. Symptoms lasted a median of 20 days (IQR, 7-56 days). PCD led to PBT discontinuation in $17 \%$ of the patients. Moreover, PBT was restarted after resolution of PCD in $43 \%$ of the patients. Only $9 \%$ of the participants had recurrent gastrointestinal symptoms.

At the time of PCD onset, the patients' median absolute neutrophil count was $3.1 \mathrm{k} / \mathrm{mm}^{3}$ (IQR, $1.4-4.2 \mathrm{k} / \mathrm{mm}^{3}$ ). Forty patients underwent $\mathrm{CT}, 64 \%$ of whom had abnormal findings. Seven patients had confirmed infections (Clostridium difficile infection in 6 and stool staphylococcus aureus infection in 1). Most patients $(59 \%)$ received treatment of $\mathrm{PCD}$ symptoms, which included antibiotics (27\%), immunosuppressants $(21 \%)$, antimotility agents $(22 \%)$, and, most frequently, intravenous fluids (51\%). The median length of PCD treatment was 14 days (IQR, 7-29 days).

The difference in the time from PBT to symptom onset according to platinum drug was not significant $(P=0.428) \quad$ (Table 2). Patients receiving oxaliplatin developed diarrhea less frequently than those receiving other PBT $(\mathrm{P}=0.02)(P=0.002)$. Also, the incidence of blood or mucous in stool $(P=0.090)$ and fever $(P=0.051)$ tended to be lower in patients receiving oxaliplatin than in those receiving the other two agents. Abdominal pain and nausea and vomiting were equally prevalent in patients receiving oxaliplatin, carboplatin, and cisplatin. The median 
duration of PCD symptoms was 7 days in patients who received oxaliplatin, whereas it was 30 days in those who received carboplatin and 25 days in patients who received cisplatin $(P=0.182)$.

Table 1. Patients' characteristics

\begin{tabular}{|c|c|}
\hline Characteristic & $\begin{array}{l}\text { Number of patients }(\%) \\
(n=86)\end{array}$ \\
\hline Median age, years (IQR) & $57(49-66)$ \\
\hline Male sex & $47(55)$ \\
\hline \multicolumn{2}{|l|}{ Race } \\
\hline Non-Hispanic White & $65(76)$ \\
\hline Black & $11(13)$ \\
\hline Other & $10(12)$ \\
\hline NSAID use & $37(43)$ \\
\hline Smoking & $48(56)$ \\
\hline Comorbidities & $52(60)$ \\
\hline Underlying IBD & $9(10)$ \\
\hline Active status & $4(5)$ \\
\hline Median ANC at time of PCD onset, $\mathrm{k} / \mathrm{mm}^{3}$ (IQR) & $3.1(1.4-4.2)$ \\
\hline \multicolumn{2}{|l|}{ Primary malignancy } \\
\hline Lung & $18(21)$ \\
\hline Gastrointestinal & $24(28)$ \\
\hline Gynecological & $16(19)$ \\
\hline Genitourinary & $9(10)$ \\
\hline Other & $19(22)$ \\
\hline Median duration of PBT, days (IQR) & $51(21-114)$ \\
\hline Median time from PBT to PCD onset, days (IQR) & $66(9-153)$ \\
\hline \multicolumn{2}{|l|}{ Platinum drug administered } \\
\hline Carboplatin & $40(47)$ \\
\hline Cisplatin & $27(31)$ \\
\hline Oxaliplatin & $19(22)$ \\
\hline \multicolumn{2}{|l|}{ Adjuvant chemotherapy } \\
\hline Taxane & $24(28)$ \\
\hline FOLFOX & $7(8)$ \\
\hline Combined regimen & $28(33)$ \\
\hline Others & $8(9)$ \\
\hline PBT stopped due to PCD & $15(17)$ \\
\hline Median duration of PCD symptoms, days (IQR) & $20(7-56)$ \\
\hline \multicolumn{2}{|l|}{ Clinical PCD symptoms } \\
\hline Diarrhea & $53(62)$ \\
\hline Abdominal pain & $41(48)$ \\
\hline Blood or mucous in stool & $37(43)$ \\
\hline Vomiting & $17(20)$ \\
\hline Fever & $5(6)$ \\
\hline \multicolumn{2}{|l|}{ Peak diarrhea grade } \\
\hline $1-2$ & $41(48)$ \\
\hline $3-4$ & $13(15)$ \\
\hline \multicolumn{2}{|l|}{ Peak colitis grade } \\
\hline $1-2$ & $68(79)$ \\
\hline 3 & $11(13)$ \\
\hline Median duration of PCD treatment, days (IQR) & $14(7-29)$ \\
\hline Hospitalization & $42(49)$ \\
\hline ICU admission & $8(9)$ \\
\hline PCD treatment & $51(59)$ \\
\hline Immunosuppressants & $18(21)$ \\
\hline Antibiotics & $23(27)$ \\
\hline Antimotility agents & $19(22)$ \\
\hline Intravenous fluids & $44(51)$ \\
\hline Colonic perforation requiring surgery & $6(7)$ \\
\hline PBT restarted after PCD resolution & $37(43)$ \\
\hline Recurrent PCD symptoms & $8(9)$ \\
\hline CT evaluation & $40(47)$ \\
\hline Abnormal findings & $27(68)$ \\
\hline \multicolumn{2}{|l|}{ Colonoscopic findings } \\
\hline Ulcer & $29(34)$ \\
\hline Inflammation & $28(33)$ \\
\hline Normal & $29(34)$ \\
\hline \multicolumn{2}{|l|}{ Colonoscopic inflammatory pattern } \\
\hline Diffuse & $20(23)$ \\
\hline Patchy & $34(40)$ \\
\hline
\end{tabular}

\begin{tabular}{ll}
\hline Characteristic & $\begin{array}{l}\text { Number of patients (\%) } \\
(\boldsymbol{n}=86)\end{array}$ \\
\hline Colitis location according to endoscopy & $22(26)$ \\
Entire colon & $28(33)$ \\
Left colon only & $6(7)$ \\
Right colon only & $65(76)$ \\
Active inflammation according to histology & $47(55)$ \\
Chronic inflammation according to histology & $14(16)$ \\
Repeat endoscopy & $5(36)$ \\
Endoscopic remission & $3(21)$ \\
Histological remission & $45(52)$ \\
Overall mortality & \multicolumn{1}{c}{ (5) } \\
\hline NSAID nonsteroidal anti-inflammatory drug, IBD inflammatory bowel disease, \\
ANC absolute neutrophil count, PCD platinum-based therapy-related diarrhea and \\
colitis. None of the mortality was related to GI toxicity.
\end{tabular}

Table 2. Patients' characteristics stratified according to platinum drug

\begin{tabular}{|c|c|c|c|c|}
\hline \multirow[b]{2}{*}{ Characteristic } & \multicolumn{3}{|c|}{ Number of patients (\%) } & \multirow[b]{2}{*}{$P$} \\
\hline & $\begin{array}{l}\text { Oxaliplatin } \\
(n=19)\end{array}$ & $\begin{array}{l}\text { Carboplatin } \\
(n=40)\end{array}$ & $\begin{array}{l}\text { Cisplatin } \\
(n=27)\end{array}$ & \\
\hline $\begin{array}{l}\text { Median time from PBT to } \\
\text { PCD onset, days (IQR) }\end{array}$ & $74(36-148)$ & $72(12-166)$ & $29(6-137)$ & 0.428 \\
\hline \multicolumn{5}{|l|}{ Clinical PCD symptoms } \\
\hline Diarrhea & $5(26)$ & $29(73)$ & $19(70)$ & 0.002 \\
\hline Abdominal pain & $6(32)$ & $22(55)$ & $13(48)$ & 0.242 \\
\hline Blood or mucous in stool & $4(21)$ & $20(50)$ & $13(48)$ & 0.090 \\
\hline Nausea and vomiting & $4(21)$ & $7(18)$ & $6(22)$ & 0.882 \\
\hline Fever & $0(0)$ & $1(3)$ & $4(15)$ & 0.051 \\
\hline $\begin{array}{l}\text { Median duration of PCD } \\
\text { symptoms, days (IQR) }\end{array}$ & $7(4-15)$ & $30(5-60)$ & $25(10-47)$ & 0.182 \\
\hline Hospitalization & $7(37)$ & $22(55)$ & $13(48)$ & 0.445 \\
\hline ICU admission & $2(11)$ & $3(8)$ & $3(11)$ & 0.864 \\
\hline $\begin{array}{l}\text { Median duration of } \\
\text { hospitalization, days (IQR) }\end{array}$ & $6(2-9)$ & $6(3-15)$ & $9(6-14)$ & 0.333 \\
\hline CT abnormality & $5(26)$ & $10(25)$ & $12(44)$ & 0.256 \\
\hline Endoscopic presentation & & & & 0.574 \\
\hline Ulcer & $6(32)$ & $11(28)$ & $12(44)$ & \\
\hline Nonulcerative inflammation & $5(26)$ & $15(38)$ & $8(30)$ & \\
\hline Normal & $8(42)$ & $14(35)$ & $7(26)$ & \\
\hline Colonoscopic colitis pattern ${ }^{b}$ & & & & 0.098 \\
\hline Diffuse & $1(5)$ & $13(33)$ & $6(22)$ & \\
\hline Patchy & $8(42)$ & $13(33)$ & $13(48)$ & \\
\hline $\begin{array}{l}\text { Active inflammation } \\
\text { according to histology }\end{array}$ & $12(63)$ & $31(78)$ & $22(81)$ & 0.508 \\
\hline $\begin{array}{l}\text { Features of chronic } \\
\text { inflammation }\end{array}$ & $6(32)$ & $24(60)$ & $17(63)$ & 0.100 \\
\hline $\begin{array}{l}\text { Colonic perforation requiring } \\
\text { surgery }\end{array}$ & $3(16)$ & $0(0)$ & $3(11)$ & 0.050 \\
\hline PCD treatment & $8(42)$ & $24(60)$ & $19(70)$ & 0.157 \\
\hline Immunosuppressants & $1(5)$ & $10(25)$ & $7(26)$ & 0.163 \\
\hline Antibiotics & $2(11)$ & $12(30)$ & $9(33)$ & 0.186 \\
\hline Antimotility agents & $5(26)$ & $5(13)$ & $9(33)$ & 0.115 \\
\hline Intravenous fluids & $7(37)$ & $22(55)$ & $15(56)$ & 0.393 \\
\hline Repeat endoscopy & $7(37)$ & $5(13)$ & $2(7)$ & 0.020 \\
\hline $\begin{array}{l}\text { PBT restarted after PCD } \\
\text { resolution }\end{array}$ & $5(26)$ & $23(58)$ & $9(33)$ & 0.037 \\
\hline Recurrent PCD symptoms & $0(0)$ & $7(18)$ & $1(4)$ & 0.046 \\
\hline
\end{tabular}

${ }^{a}$ Available for 27 patients. ${ }^{b}$ Available for 54 patients.

Participants who presented with diarrhea $(P<0.001)$, vomiting $(P<0.006)$, or abdominal pain $(P<0.001)$ were more likely to undergo treatment of PCD symptoms than were those who did not receive treatment (Table 3). Those who presented with fever $(P=0.081)$ or blood or mucous in stool $(P=0.077)$ were only somewhat more likely to receive treatment. Recurrent PCD was equally present in the treatment and nontreatment groups. The duration of PCD 
symptoms was similar in these two groups $(P=0.247)$. The use of different treatment regimens, including immunosuppressants, intravenous fluids, antibiotics, and antimotility agents, was equally prevalent in participants who received oxaliplatin, carboplatin, and cisplatin.

\section{Endoscopy and Histology}

Colonoscopic findings were roughly evenly distributed: normal colonic tissue in $34 \%$ of the participants, mucosal ulceration in $34 \%$, and nonulcerative inflammation in 33\% (Fig. 2). Regarding the pattern of endoscopic inflammation, $40 \%$ of colonoscopies demonstrated patchy inflammation, whereas $23 \%$ demonstrated diffuse inflammation. Thirty-three percent of abnormal endoscopic findings were in the left colon only, and 7\% were in the right colon only. Only $26 \%$ of the study participants had involvement of the entire colon (Table 1).

In terms of ulcers, nonulcerative inflammation, and normal colonic tissue, we observed no significant endoscopic differences regarding these features between the treatment and nontreatment groups. Also, the platinum drug received did not correlate with endoscopic findings. However, diffuse inflammation was most common in carboplatin recipients $(50 \%)$ and least common in oxaliplatin recipients $(11 \%)$. The trend for patchy inflammation was reversed, as it was most common in oxaliplatin recipients $(89 \%)$ and least common in carboplatin recipients $(50 \%)$. Of note, this association was only partially significant and appeared to be secondary to the varying presence of inflammation in the oxaliplatin group $(P=0.098)$ (Tables 2 and 3$)$.

Histologically, inflammation was categorized as active or chronic. Seventy-six percent of the participants had histologies demonstrating active inflammation, whereas $55 \%$ had chronic inflammation. We found active and chronic inflammation patterns equally in the treatment and nontreatment groups. The presence of active or chronic inflammation did not differ according to type of PBT (Table 3).

\section{Clinical Outcomes}

Forty-nine percent of the patients had to be hospitalized, 9\% needed ICU unit admission, and 7\% had a colonic perforation requiring surgery. Three of these 6 patients with complications received bevacizumab as part of adjuvant chemotherapy. Complications were less likely in those who received carboplatin than in those given the other two platinum drugs $(P=0.05)$. Recurrent gastrointestinal symptoms occurred in $25 \%$ of patients who resumed PBT after PCD onset $(n=37)$. Recurrent PCD was absent from oxaliplatin recipients and most common in carboplatin recipients $(18 \% ; P=0.046)$.

Restarting PBT after the occurrence of PCD was most common in the carboplatin group (58\%) followed by the cisplatin (33\%) and oxaliplatin (26\%) groups $(P=0.037)$. Only $16 \%$ of the participants underwent repeat endoscopy. We noted endoscopic remission in $36 \%$ of the study participants and histological remission in $25 \%$ of them. Furthermore, the treatment and nontreatment groups were equally likely to receive PBT after occurrence of PCD or to undergo repeat endoscopy. Repeat endoscopy was most common in the oxaliplatin group and least common in the cisplatin group $(P=0.02)$.
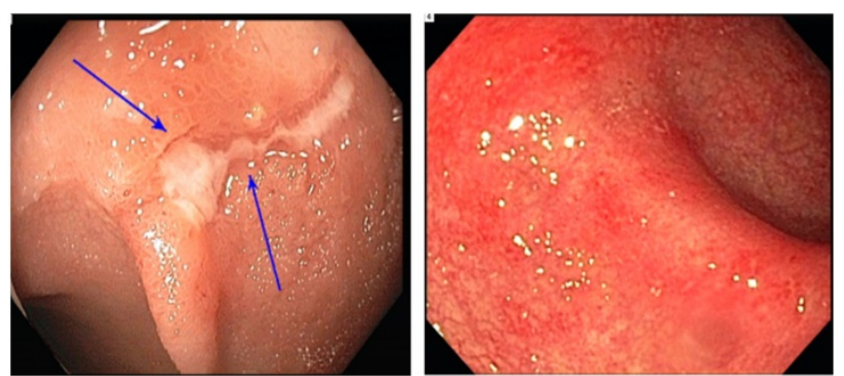

Figure 2. Endoscopy presentation of platinum-related colitis. Superficial ulcers (left); and diffuse erythema and edema (right).

Table 3. Patients' characteristics stratified according to PCD treatment

\begin{tabular}{|c|c|c|c|}
\hline \multirow[t]{2}{*}{ Characteristic } & \multicolumn{2}{|c|}{ Number of patients (\%) } & \multirow[t]{2}{*}{$P$} \\
\hline & $\begin{array}{l}\text { Treatmenta } \\
(n=51)\end{array}$ & $\begin{array}{l}\text { No treatment } \\
(n=35)\end{array}$ & \\
\hline Platinum drug & & & 0.157 \\
\hline Carboplatin & $24(47)$ & $16(46)$ & \\
\hline Cisplatin & $19(37)$ & $8(23)$ & \\
\hline Oxaliplatin & $8(16)$ & $11(31)$ & \\
\hline \multicolumn{4}{|l|}{ Clinical PCD symptoms } \\
\hline Diarrhea & $41(80)$ & $12(34)$ & $<0.001$ \\
\hline Abdominal pain & $33(65)$ & $8(23)$ & $<0.001$ \\
\hline Blood or mucous in stool & $26(51)$ & $11(31)$ & 0.081 \\
\hline Vomiting & $15(29)$ & $2(6)$ & 0.006 \\
\hline Fever & $5(10)$ & $0(0)$ & 0.077 \\
\hline $\begin{array}{l}\text { Median duration of PCD symptoms, days } \\
\text { (IQR) }\end{array}$ & $28(9-56)$ & $12(3-53)$ & 0.361 \\
\hline $\begin{array}{l}\text { Median duration of hospitalization, days } \\
\text { (IQR) }\end{array}$ & $8(3-14)$ & $5(3-9)$ & 0.247 \\
\hline CT abnormality & $21(41)$ & $6(17)$ & 0.163 \\
\hline Endoscopic presentation & & & 0.754 \\
\hline Ulcer & $18(35)$ & $11(31)$ & \\
\hline Nonulcerative inflammation & $15(29)$ & $13(37)$ & \\
\hline Normal & $18(35)$ & $11(31)$ & \\
\hline $\begin{array}{l}\text { Active inflammation according to } \\
\text { histology }\end{array}$ & $42(82)$ & $23(66)$ & 0.390 \\
\hline Features of chronic inflammation & $31(61)$ & $16(43)$ & 0.367 \\
\hline Colonic perforation requiring surgery & $4(8)$ & $2(6)$ & 1.000 \\
\hline PBT restarted after PCD resolution & $22(43)$ & $15(43)$ & 1.000 \\
\hline Repeat endoscopy & $7(14)$ & $7(20)$ & 0.555 \\
\hline Recurrent PCD symptoms & $6(12)$ & $2(6)$ & 0.464 \\
\hline
\end{tabular}

aTreatment of PCD included intravenous fluids, antibiotics, immunosuppressants, and antimotility agents. ${ }^{\mathrm{b}}$ Available for 27 patients. 


\section{Discussion}

This retrospective study sheds light on the importance of the clinical, endoscopic, and histological characteristics of PCD and its outcomes. The incidence of PCD after PBT was very low, but PCD can lead to significant morbidity, such as colonic perforation. Treatment with carboplatin or cisplatin was more likely to cause diarrhea and blood or mucous in stool than was treatment with oxaliplatin. Although information regarding PCD in the literature is scarce, a significant proportion of our cohort received various types of nonspecific PCD treatment.

Authors have reported that the incidence rate for diarrhea and abdominal pain associated with PBT ranges from $30 \%$ to $50 \%$ in patients.[11] However, the incidence of endoscopy-confirmed colitis has yet to be reported. In our cohort, the incidence of PCD was very low, as we included only patients who underwent colonoscopic evaluation. Several case reports and studies demonstrated gastrointestinal side effects of PBT, especially that with carboplatin and cisplatin.[12-17] They described PCD as a form of ischemic colitis, IBD, infectious colitis, or neutropenic enterocolitis given the overlapping presentations. Therefore, to account for possible confounding factors, we included only patients who underwent colonoscopy and biopsy. In our cohort, 4 out of 9 patients had active status of IBD at the time of PBT. Confounding factors from active IBD symptoms or exacerbation with PCD need to be considered. In addition, despite that the average absolute neutrophil count in the patients was in the normal range (perhaps because symptom onset occurred at a median of 66 days after PBT began), frequently experienced neutropenic episodes expected during PBT treatment could still have had occurred, affecting colitis presentation. With these differential diagnoses for colitis, endoscopy and histology evaluation will provide valuable information to clarify the etiology of colitis and guide subsequent management.

Our findings bring up the question of whether to treat PCD upon initial presentation. Given that a minority of patients could have bacteremia or a gastrointestinal infection, we must determine whether antibiotics should be given immediately in cases of suspected colitis. Standard of practice dictates that when a cancer patient presents with nonspecific abdominal or constitutional symptoms, broadspectrum antibiotics are given. However, indiscriminate use of antibiotics can lead to an increase in the incidence of gastrointestinal symptoms. Some PCD patients are not immunocompromised or febrile, and so their PCD is best managed via expectant care (i.e., intravenous fluids, antimotility agents).

Analysis of patient characteristics demonstrated that in the $49 \%$ of participants who needed hospitalization, the median duration of hospitalization ranged from 5 to 9 days. Of note, PCD symptoms lasted an average of 20 days, indicating that they were mild enough to not always necessitate hospitalization. This finding also demonstrates that treatment of colitis symptoms, which was primarily conservative, could have continued in the outpatient setting. Unsurprisingly, PCD symptoms were manageable enough that PBT had to be stopped in only $17 \%$ of cases.

With all three platinum drugs, we found that patients with PCD presented with nonspecific abdominal pain and nausea and vomiting. In light of the fact that $28 \%$ of the patients had primary gastrointestinal malignancies, such nonspecific symptoms could be attributed to multiple causes. However, diarrhea and blood or mucous in stool were equally common in the carboplatin and cisplatin groups but rare in the oxaliplatin group. Therefore, when evaluating gastrointestinal symptoms in patients with a history of PBT exposure, an important step is to note that 1) oxaliplatin recipients present with mild, nonspecific symptoms and 2) symptoms may not be attributed to malignancy, infection, or graft-versus-host disease.

Unsurprisingly, the presence of PCD symptoms was significantly associated with treatment of these symptoms. However, participants with longer symptom durations were not more likely to receive PCD treatment. This demonstrates that PCD symptoms were likely self-limited. However, it may also demonstrate that some patients have baseline intermittent gastrointestinal symptoms (due to either medications or malignancy) that are unrelated to PCD.

Roughly half of the patients $(47 \%)$ underwent CT. Also, two thirds of the patients had an abnormal endoscopy, demonstrating that an unremarkable CT scan does not negate the need for a colonoscopy. Authors have reported undefined imaging and endoscopic findings indicative of colitis in other studies, as well.[18] Only 7\% of patients had PCD exclusively in the right colon. Therefore, sigmoidoscopy rather than the more invasive colonoscopy could be considered in patients with suspected colitis in whom colonoscopy may be risky. The presence of mucosal ulceration or nonulcerative inflammation according to endoscopy did not correlate with an increase in the administration of treatment of PCD, and neither did active or chronic inflammation according to histological examination. This is likely due to the fact that patients received treatment based on clinical PCD symptoms alone and not endoscopic findings. Again, the clinical picture is 
not predictive of endoscopic abnormalities, and both should be considered in evaluating a patient with suspected PCD.

Carboplatin recipients were the most likely to have PBT restarted after resolution of PCD and the least likely to have colitis complications. In the future, endoscopy in carboplatin recipients may be safer than that in patients receiving other platinum drugs. In contrast, oxaliplatin recipients had the highest prevalence of colitis complications but the fewest recurrent gastrointestinal symptoms. Clearly, initial clinical presentation does not correlate with future symptoms in patients with PCD. To minimize these side effects in the future, new classes of platinum agents are being developed (e.g., nedaplatin, lobaplatin, heptaplatin), but they have limited regional approval [19].

This study has some limitations. First, the retrospective design limited our ability to obtain detailed and accurate clinical information regarding medications used and their duration of use. Second, the absence of treatment standardization and uncertainty in PCD diagnosis led to arbitrary treatment approaches that may not have been based on evidence-based medicine. Third, including patients who underwent colonoscopic and histological evaluation could have precluded some cases of PCD. Fourth, despite being the largest study on this topic, the small sample size underpowers any meaningful analyses.

\section{Conclusions}

PCD is rare, but in some patients, it can lead to severe complications requiring surgical intervention. The clinical presentation of PCD is nonspecific and can be confused with other etiologies of colitis. Still, endoscopic and histological findings can increase the degree of certainty of diagnosis and lead to prompt treatment to prevent adverse consequences. Resumption of PBT after PCD resolution results in mild gastrointestinal symptom recurrence in $25 \%$ of patients, which can be managed expectantly. Future studies investigating the appropriate treatment approaches for PCD are needed.

\section{Acknowledgements}

Medical editing of this paper was provided by the Department of Scientific Publications at MD Anderson Cancer Center.

\section{Competing Interests}

The authors have declared that no competing interest exists.

\section{References}

1. Chen, X., Y. Wu, H. Dong, et al. Platinum-based agents for individualized cancer treatment. Current molecular medicine, 2013. 13(10): 1603-12.

2. [Internet] WHO Model Lists of Essential Medicines. Accessed online January 11, 2019. https://www.who.int/medicines/publications/essentialmedicines/ en/

3. Puisset, F., A. Schmitt, and E. Chatelut, Standardization of chemotherapy and individual dosing of platinum compounds. Anticancer research, 2014. 34(1): 465-70.

4. Pfisterer, J. and J.A. Ledermann, Management of platinum-sensitive recurrent ovarian cancer. Seminars in oncology, 2006. 33(2 Suppl 6): S12-6.

5. McQuade, R.M., V. Stojanovska, J.C. Bornstein, et al. Colorectal Cancer Chemotherapy: The Evolution of Treatment and New Approaches. Current medicinal chemistry, 2017. 24(15): 1537-1557.

6. Andre, T, A de Gramont, D. Vernerey, et al. Adjuvant Fluorouracil, Leucovorin, and Oxaliplatin in Stage II to III Colon Cancer: Updated 10-Year Survival and Outcomes According to BRAF Mutation and Mismatch Repair Status of the MOSAIC Study. Journal of clinical oncology: official journal of the American Society of Clinical Oncology, 2015. 33(35): 4176-87.

7. Matsuura, T. and I. Otsuka, [Effectiveness of Carboplatin plus Taxane Chemotherapy for Advanced or Recurrent Uterine Serous Carcinoma]. Gan to kagaku ryoho. Cancer \& chemotherapy, 2016. 43(11): 1401-1404.

8. Tharavichitkul, E., V. Lorvidhaya, P. Kamnerdsupaphon, et al. Combined chemoradiation of cisplatin versus carboplatin in cervical carcinoma: a single institution experience from Thailand. BMC cancer, 2016. 16: 501.

9. Oronsky, B., S. Caroen, A. Oronsky, et al. Electrolyte disorders with platinum-based chemotherapy: mechanisms, manifestations and management. Cancer chemotherapy and pharmacology, 2017. 80(5): 895-907.

10. Charlson, M.E, P. Pompei, K.L. Ales, et al. A new method of classifying prognostic comorbidity in longitudinal studies: development and validation. Journal of chronic diseases, 1987. 40(5): 373-83.

11. Cassidy J., Misset J.L. Oxaliplatin-related side effects: characteristics and management, 2002. 29(5 suppl 15):11-20.

12. Osumi, H., M. Ozaka, H. Ishii, et al. Severe ischemic colitis after treatment of bile-duct cancer using gemcitabine and cisplatin. Japanese journal of clinical oncology, 2015. 45(4): 402-3.

13. Fujita, K., Y. Mizumoto, K. Moriyoshi, et al. Acute onset of ulcerative colitis during chemoradiotherapy for anaplastic lymphoma kinase-positive lung adenocarcinoma. Respirology case reports, 2018. 6(2): e00288.

14. Ji, E.H., Y.M. Kim, S.J. Kim, et al. A case of typhlitis developed after chemotherapy with irinotecan and Cisplatin in a patient with small cell lung carcinoma. Tuberculosis and respiratory diseases, 2012. 73(5): 288-91.

15. Elsayed, A.G., R. Srivastava, T. Pacioles, et al. Ischemic Colitis Associated with Paclitaxel and Carboplatin Combination. Case reports in oncology, 2017. 10(2): 689-693.

16. Allen, V.A, K.J. Manahan, and J.P. Geisler, Recurrent Pseudomembranous Colitis in an Ovarian Cancer Patient Undergoing Carboplatin Chemotherapy. Case reports in obstetrics and gynecology, 2016. 2016: 7540302.

17. Tashiro, M., I. Yoshikawa, K. Kume, et al. Ischemic colitis associated with paclitaxel and carboplatin chemotherapy. The American journal of gastroenterology, 2003. 98(1): 231-2.

18. Kuebler, J.P., L. Colangelo, M.J. O'Connell, et al. Severe enteropathy among patients with stage II/III colon cancer treated on a randomized trial of bolus 5-fluorouracil/leucovorin plus or minus oxaliplatin: a prospective analysis. Cancer, 2007. 110(9): 1945-50

19. Dilruba, S. and G.V. Kalayda, Platinum-based drugs: past, present and future. Cancer chemotherapy and pharmacology, 2016. 77(6): 1103-24. 